\title{
Mortalidad por Causas Externas en Estado de Paraná y Municipio de Foz de Iguazú, 1996 a 2016
}

\author{
Mortality from External Causes in the State of Paraná and the Municipality of \\ Foz de Iguazú, 1996 to 2016 \\ Derlis Gustavo Adolfo Duarte-Zoilán (1) 1, Carmen Justina Gamarra (1)2
}

${ }^{1}$ Ministerio de Salud Pública y Bienestar Social, Asunción, Paraguay.

¿Universidad Federal de Intregración Latino-Americana, Brasil.

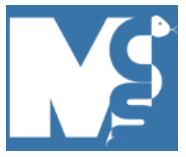

Recibido: $18 / 10 / 2021$

Revisado: 01/12/2021

Aceptado:10/12/2021

\section{Autor correspondiente}

Derlis Duarte-Zoilán

Ministeiro de Salud Pública y

Bienestar Social, Asunción,

Paraguay

derliszoilan@gmail.com

\section{Conflictos de interés}

El autor declara no poseer conflictos de interés.

\section{Fuente de financiación}

El autor no recibió apoyo financiero para la investigación, autoría y/o publicación de este artículo.

Este artículo es publicado bajo una licencia de Creative Commons

Reconocimiento 4.0 Internacional.

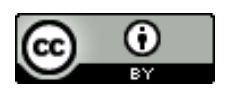

\section{RESUMEN}

Introducción: Las Causas Externas (CE) de morbilidad y mortalidad son identificadas como lesiones intencionales (homicidios, violencia, suicidios, privación o negligencia) y lesiones no intencionales (accidentes de transportes, ahogamientos, caídas, quemaduras, intoxicaciones accidentales, complicaciones de asistencias médicas, entre otros). Objetivo: analizar los casos de mortalidad por causas externas en el Estado de Paraná y Municipio de Foz de Iguazú en el periodo de 1996 a 2016. Metodología: Se realizó un estudio cuantitativo, observacional, ecológico descriptivo, retrospectivo donde se analizó los óbitos por causas externas en el Estado de Paraná y Municipio de Foz de Iguazú en el periodo de 1996 a 2016 entre hombres y mujeres. A partir de datos secundarios disponibles en el Departamento de Informática del Sistema Único de Salud (DATASUS). Resultados: Las tendencias de mortalidad por causas externas en el periodo de 1996 a 2016 en el Estado de Paraná fue estable y en el Municipio de Iguazú mostró disminución; el promedio en las tasas de mortalidad de 8,7 y 12,4 por 10.000 habitantes para Paraná y Foz de Iguazú respectivamente. Las causas más importantes de muerte en el inicio del estudio fueron Agresiones y Accidentes de Tránsitos tanto para Paraná como para Foz de Iguazú, con mayor índice en las edades de 15 a 29 años en ambos sexos, con diferencia al final del estudio donde la primera causa de muerte en hombres es agresiones con índices mayores en edades de 15 a 29 años y otras lesiones por accidentes en mujeres de 60 años y más. Conclusión: En los 21 años de estudios muestran que los hombres representan el mayor índice de óbitos por causas externas, las tasas de mortalidad por óbitos de causas externas siguen siendo alarmantes para el Estado de Paraná y principalmente para Foz de Iguazú, afectando mayormente a la población joven masculina en edad reproductiva y trabajadora.

Palabras clave: Mortalidad; Causas Externas; Salud Colectiva; Epidemiologia, salud pública.

\section{ABSTRACT}

Introduction: External Causes (EC) of morbidity and mortality are identified as intentional injuries (homicides, violence, suicides, deprivation or negligence) and unintentional injuries (transport accidents, drowning, falls, burns, accidental poisoning, complications of medical assistance, among others). Objective: to analyze the cases of mortality due to external causes in the State of Paraná and Municipality of Foz do Iguaçu in the period from 1996 to 2016. Methodology: a quantitative, observational, descriptive ecological, retrospective study was conducted where the deaths due to external causes were analyzed in the State of Paraná and Municipality of Foz do Iguaçu in the period from 1996 to 2016 among men and women. From secondary data available in the Informatics Department of the Unified Health System (DATASUS). Results: The trends in mortality from external causes in the period from 1996 to 2016 in the State of Paraná was stable and in the Municipality of Iguaçu showed decrease; the average in mortality rates of 8.7 and 12.4 per 10,000 inhabitants for Paraná and Foz do Iguaçu respectively. The most important causes of death at the beginning of the study were assaults and traffic accidents for both Paraná and Foz do Iguaçu, with higher rates in ages 15 to 29 years in both sexes, with a difference at the end of the study where the first cause of death in men is assaults with higher rates in ages 15 to 29 years and other injuries due to accidents in women aged 60 years and over. Conclusion: In the 21 years of studies, men represent the highest rate of deaths due to external causes. Mortality rates for deaths due to external causes continue to be alarming for the State of Paraná and mainly for Foz do Iguaçu, affecting mostly the young male population of reproductive and working age. 


\section{INTRODUCCIÓN}

Las Causas Externas (CE) de morbilidad y mortalidad son identificadas como lesiones intencionales (homicidios, violencia, suicidios, privación o negligencia) y lesiones no intencionales (accidentes de transportes, ahogamientos, caídas, quemaduras, intoxicaciones accidentales, complicaciones de asistencias médicas, entre otros) (1).

La mortalidad por CE es reconocida como un grave problema de salud pública (2). La Organización Mundial de la Salud (OMS) afirma que estas causas son responsables de 5 millones de muertes en el mundo; 2 millones más que el virus de la inmunodeficiencia humana (VIH), paludismo y tuberculosis juntos en el 2012 (3). En el Brasil, en el 2016, las causas externas correspondieron la cuarta mayor causa de muerte en el país el $12 \%$ del total de los óbitos (2).

Los Estados con mayores índices por CE en Brasil son San Paulo, Río de Janeiro, Minas Gerias y Bahía, estando Paraná en la quinta posición y primero de la Región Sur con 13,27\% de los óbitos, constituyendo la tercera causa de muerte más importante, con diferencia al municipio de Foz de Iguazú que ocupa el primer lugar con $23,82 \%$ de los óbitos (4). Solo en el 2016 el $82 \%$ de los óbitos por CE corresponde al sexo masculino (2).

Según la Organización Panamericana de la Salud (OPS) los factores externos constituyen la principal causa de pérdida de años de vida (AVPP) en dos tercios de las naciones americanas, siendo los accidentes de transporte, homicidios y suicidios los que figuran entre las cinco principales de causas de muertes prematuras (5).

Las CE son responsables en gran parte de las internaciones hospitalarias en Brasil y, a pesar de presentar un tiempo menor de internación, representan un impacto más significativo para los recursos públicos de salud del observado por causas naturales (6). En el 2017 fueron registrados 1071711 internaciones hospitalarias por causas externas, lo que representa $9,9 \%$ de todas las internaciones realizadas en los servicios propios y tercerizados del Sistema Único de Salud (SUS) (2).

En el 2010 el Estado de Paraná tuvo un gasto en reales (R\$) de 83741809,39 por internaciones en el SUS por causas externas (7). Además, imponen gran demanda a los servicios de salud, ya que es al sistema de salud que muchas de las víctimas se dirigen en busca de atención de emergencia, asistencia especializada, rehabilitación física y psicológica. En ese sentido, las causas externas constituyen un tema relevante y su prevención se ha vuelto una prioridad en el área de la Salud $(6,8)$. Las CE también tienen un importante impacto sobre la esperanza de vida al nacer del brasilero. Estas CE representan consecuencias económicas y sociales que se traducen en días de ausencia en el trabajo, costo para el sistema de salud, demanda a los servicios sociales, pérdida de vida productiva por muerte. Asimismo, produce daños mentales y emocionales, muchas veces invisibles e inmensurables, tanto para las víctimas como para las familias (2).

El estudio de la mortalidad por CE es importante para obtener un análisis de la situación en relación a las causas más importantes por óbitos no esperados, generando así conocimiento de su magnitud y evolución, pudiendo contribuir para el desarrollo de acciones y planeamientos en salud.

El presente trabajo tiene como objetivo analizar los casos de mortalidad por causas externas en el Estado de Paraná y Municipio de Foz de Iguazú en el periodo de 1996 a 2016.

\section{METODOLOGÍA}

Fue realizado un estudio cuantitativo, observacional, ecológico descriptivo y retrospectivo, como ámbito geográfico de estudio fueron tomadas el Estado de Paraná y el Municipio de Foz de Iguazú, durante el periodo de 1996 a 2016. Se analizaron los datos de óbitos a partir del año 1996 ya que anterior a estos años corresponde a otra nomenclatura por causas externas y no se encuentran codificados en el Capítulo 10 de la Clasificación Estadísticas Internacional de Enfermedades y Problemas Relacionados a la Salud Décima Revisión (CIE-10). Los Datos sobre óbitos fueron obtenidos del Sistema de Información de Mortalidad (SIM) y los Demográficos del Instituto Brasilero de Geografía y Estadística (IBGE).

Las variables incluidas en el estudio fueron: año y localidad de ocurrencia del óbito, sexo, causa básica de muerte y la edad por grupo etario. Se seleccionaron los registros cuya causa básica de muerte en el SIM estaban clasificados en el Capítulo XX del (CIE-10), de acuerdo a los siguientes agrupamientos: Total de causas externas (V01-Y84); Accidentes de transporte terrestre (V01-V89) ; Otros tipos de accidentes (W00 X59) como: Caídas, Ahogamiento, Electrocución, Envenenamiento, Quemaduras; Lesiones autoprovocadas intencionalmente $(X 60-X 84)$ que serán denominadas en este estudio como suicidios; Agresiones (X85-Y09): homicidios; otras causas externas (Y10 - Y84) como eventos cuja intención es indeterminada, Intervención legal y operaciones de guerra, Complicaciones de asistencia médica y 
quirúrgica.

Para el análisis de los datos fueron calculadas las tasas de Mortalidad ajustadas por la población padrón de la OMS, Mortalidad Proporcional y promedio de las tasas, para el análisis de la tendencia de mortalidad ajustada se optó por el modelo de regresión lineal teniendo en cuenta coeficiente de beta y valor de $p$, que permitieron la estimación de los parámetros de regresión visual, y su clasificación en aumento, reducción o estabilidad.

Para los procedimientos estadísticos fueron utilizados los programas EPI - Info 7 (Programa de dominio público) y Microsoft Excel 2010. El estudio envolvió apenas la colecta y análisis documental de datos secundarios y de literaturas disponibles, siendo enviado al Consejo Nacional de Salud (CONEP) del Ministerio de Salud y probado por resolución: 475.925 y Certificado de Presentación para Apreciación Ética (CAAE) 22459713.3.0000.5230.

\section{RESULTADOS}

En el periodo estudiado entre 1996 y 2016, en el Estado de Paraná fueron registrados 172909 óbitos, de los cuales el $82 \%$ ( $n=141926$ ) fueron del sexo masculino. En el Municipio de Foz de Iguazú fueron registrados 6 881 óbitos, $87 \%$ ( $n=5927$ ) en hombres. El promedio de óbitos fue de 8,7 y de 12,4 óbitos por 10000 habitantes en el Estado de Paraná y Foz de Iguazú.

Se puede observar que, entre 1996 y 2016 en el Estado de Paraná existió un comportamiento estable en la Tendencia de Mortalidad. El sexo masculino presentó una tendencia más elevada en relación a las mujeres (promedio de 13,4 a 2,9 óbitos por 10000 habitantes). En tanto, en el Municipio de Foz de Iguazú, fue observada una disminución en la tendencia, en ambos sexos con promedios de 21,5 y 3,8 por 10000 habitantes respectivamente (Paraná, Total: 0,006; Hombres: 0,019; Mujeres: -0,009), (Foz, Total: -0,17; Hombres: -0,319; Mujeres: -0,319) (Figura 1 y Figura 2).

Los coeficientes de regresión de las tasas de mortalidad por causas externas por sexo en Foz de Iguazú y Paraná de 1996 a 2016 se aprecian en la Tabla 1.

FIGURA 1: TASA DE MORTALIDAD POR CAUSAS EXTERNAS POR 10000 HABITANTES POR SEXO Y TOTAL EN EL ESTADO DE PARANÁ, 1996 A 2016.

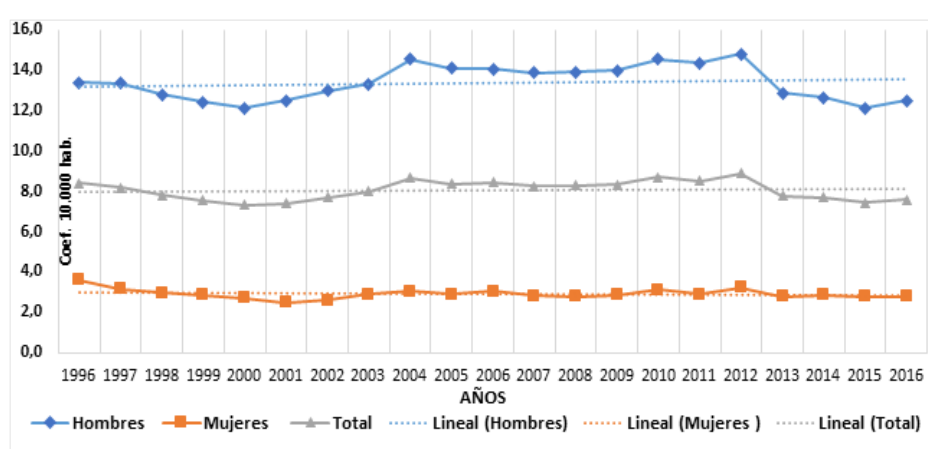

FIGURA 2: TASA DE MORTALIDAD POR CAUSAS EXTERNAS POR 10.000 HABITANTES POR SEXO Y TOTAL EN EL MUNICIPIO DE FOZ DE IGUAZÚ, 1996 A 2016.

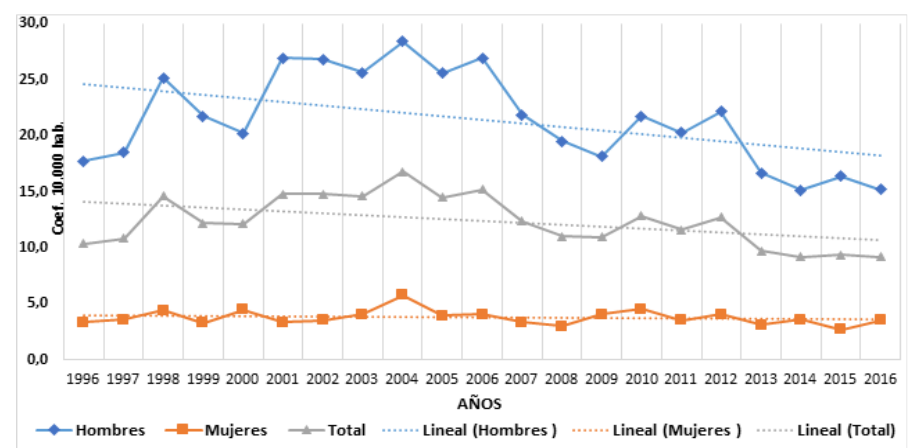


TABLA 1: COEFICIENTES DE REGRESIÓN DE LAS TASAS* DE MORTALIDAD POR CAUSAS EXTERNAS, POR SEXO. FOZ DE IGUAZÚ Y PARANÁ, 1996 A 2016.

\begin{tabular}{cccccc}
\hline \multirow{2}{*}{ Región } & Grupo & Coeficiente $\boldsymbol{B}$ & $\boldsymbol{P}$ valor & $\boldsymbol{R}^{2}$ & Tendencia \\
\hline \multirow{2}{*}{ Foz de Iguazú } & Hombres & $-0,319$ & 0,03118 & 0,22 & Disminuye \\
& Mujeres & $-0,022$ & 0,375794 & 0,04 & Estable \\
& Total & $-0,17$ & 0,032141 & 0,22 & Disminuye \\
\hline \multirow{2}{*}{ Paraná } & Hombres & 0,019 & 0,559874 & 0,02 & Estable \\
& Mujeres & $-0,009$ & 0,326366 & 0,05 & Estable \\
& Total & 0,006 & 0,717992 & 0,01 & Estable \\
\hline
\end{tabular}

*Tasas de mortalidad por causas externas ajustadas por edad según poblacional padrón Mundial de la OMS.

En cuanto a la mortalidad proporcional de los grupos de causas externas más importantes de los 21 años de estudio, según figura 3 y 4 se observa que en el Estado de Paraná la primera causa de muerte con el $36,8 \%$ corresponde a ATT, agresiones $32,6 \%$ y otros tipos de accidentes $18,8 \%$, del total de los óbitos del periodo estudiado. Con relación al Municipio de Foz de Iguazú las agresiones representan la primera causa de muerte con el $56,3 \%$, ATT el $25,5 \%$ y otros tipos de accidentes 10,9 de los óbitos (Figura 3). En la Figura 4 se aprecia Mortalidad proporcional por grandes grupos de causas externas en el Municipio de Foz de Iguazú, 1996 a 2016.

FIGURA 3: MORTALIDAD PROPORCIONAL POR GRANDES GRUPOS DE CAUSAS EXTERNAS EN EL ESTADO DE PARANÁ, 1996 A 2016.

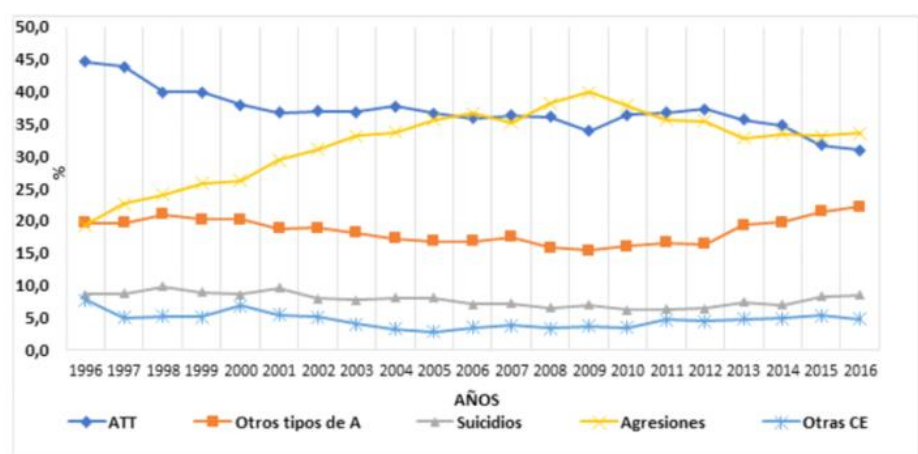

FIGURA 4: MORTALIDAD PROPORCIONAL POR GRANDES GRUPOS DE CAUSAS EXTERNAS EN EL MUNICIPIO DE FOZ DE IGUAZÚ, 1996 A 2016.

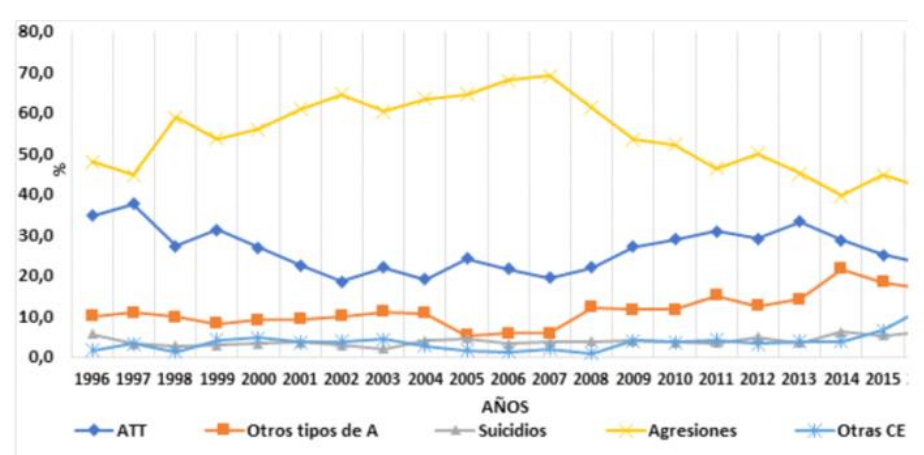

A partir del coeficiente de regresión de los grupos de causas externas en los años de estudios se observa que en el Estado de Paraná tanto como en el Municipio de Foz de Iguazú, los casos de accidentes de tránsitos han disminuido, con relación a otros tipos de accidentes en Paraná la tendencia disminuye, con diferencia de Foz de Iguazú que está en aumento. Agresiones en el Estado de Paraná aumenta con diferencia de Foz de Iguazú que disminuye. Suicidios y Otras causas externas en Paraná está en disminución, con relación de Foz de Iguazú que va en aumento (Tabla 2). 
TABLA 2: COEFICIENTES DE REGRESIÓN DE LAS TASAS DE MORTALIDAD PROPORCIONAL POR CAUSAS EXTERNAS, POR GRUPOS DE CAUSAS. FOZ DE IGUAZÚ Y PARANÁ, 1996 A 2016.

\begin{tabular}{|c|c|c|c|c|c|}
\hline Región & Causas Externas & Coeficiente $\boldsymbol{B}$ & P valor & $R^{2}$ & Tendencia \\
\hline \multirow[t]{5}{*}{ Foz de Iguazú } & ATT* & $-0,117$ & 0,555291 & 0,02 & Disminuye \\
\hline & Otros Tipos A. ${ }^{* *}$ & 0,435 & 0,001149 & 0,43 & Aumenta \\
\hline & Suicidios & 0,095 & 0,01657 & 0,27 & Aumenta \\
\hline & Agresiones & $-0,595$ & 0,064915 & 0,17 & Disminuye \\
\hline & Otras $\mathrm{CE}^{* * *}$ & 0,182 & 0,030779 & 0,22 & Aumenta \\
\hline \multirow[t]{5}{*}{ Paraná } & ATT* $^{*}$ & $-0,437$ & 0,000002 & 0,7 & Disminuye \\
\hline & Otros Tipos A. ${ }^{* *}$ & $-0,04$ & 0,588126 & 0,02 & Disminuye \\
\hline & Suicidios & $-0,106$ & 0,002572 & 0,39 & Disminuye \\
\hline & Agresiones & 0,654 & 0,000134 & 0,54 & Aumenta \\
\hline & Otras $\mathrm{CE}^{* * *}$ & $-0,071$ & 0,100607 & 0,14 & Disminuye \\
\hline
\end{tabular}

** Otros tipos de Accidentes (Caídas, Ahogamiento, Electrocución, Envenenamiento, Quemaduras)

*** Otras Causas Externas (Eventos cuja intención es indeterminada, Intervención legal y operaciones de guerra, Complicaciones de asistencia médica y quirúrgica).

En la Tabla 3 al inicio del estudio se observa que en el Estado de Paraná la causa de muerte más importante en los dos sexos es Accidentes de tránsitos terrestres (ATT), Foz de Iguazú, agresiones corresponde el 55,2\% de los óbitos en el sexo masculino y ATT el 61,5\% de los óbitos en el sexo femenino, cabe importante mencionar que en el año 1996 en los dos sexos el grupo etario más afectado es el de 15 a 29 años de edad. La Tabla 4 del año 2016, tanto en el Estado de Paraná como en el Municipio de Foz de Iguazú en el sexo masculino la causa más importante de muerte es agresiones en las edades de 15 a 29 años y en el sexo femenino es otras lesiones en edades de 60 años y más.

TABLA 3: MORTALIDAD PROPORCIONAL DE GRUPOS DE CAUSAS EXTERNAS POR SEXO Y GRUPO ETARIO, EN EL ESTADO DE PARANÁ Y MUNICIPIO DE FOZ DE IGUAZÚ, 1996.

\begin{tabular}{|c|c|c|c|c|c|c|c|c|c|c|c|c|}
\hline \multicolumn{13}{|c|}{ ESTADO DE PARANÁ } \\
\hline \multicolumn{13}{|c|}{ MASCULINO } \\
\hline & \multicolumn{2}{|c|}{ O a 14} & \multicolumn{2}{|c|}{15 a 29} & \multicolumn{2}{|c|}{30 a 44} & \multicolumn{2}{|c|}{45 a 59} & \multicolumn{2}{|c|}{$>60$} & \multicolumn{2}{|c|}{ Total } \\
\hline Tipos de Accidentes & $N$ & $\%$ & $N$ & $\%$ & $N$ & $\%$ & $N$ & $\%$ & $N$ & $\%$ & $N$ & $\%$ \\
\hline ATT & 198 & 8,0 & 839 & 34,1 & 749 & 30,4 & 418 & 17,0 & 257 & 10,4 & 2461 & 44,4 \\
\hline Otras lesiones & 214 & 21,6 & 258 & 26,0 & 222 & 22,4 & 140 & 14,1 & 159 & 16,0 & 993 & 17,9 \\
\hline Suicidios & 8 & 1,7 & 171 & 35,8 & 142 & 29,8 & 71 & 14,9 & 85 & 17,8 & 477 & 8,6 \\
\hline Agresiones & 18 & 1,5 & 597 & 49,5 & 378 & 31,4 & 157 & 13,0 & 55 & 4,6 & 1205 & 21,8 \\
\hline Otras Causas Externas & 33 & 8,2 & 154 & 38,1 & 121 & 30,0 & 61 & 15,1 & 35 & 8,7 & 404 & 7,3 \\
\hline Total & 471 & 8,5 & 2019 & 36,4 & 1612 & 29,1 & 847 & 15,3 & 591 & 10,7 & 5540 & 100 \\
\hline \multicolumn{13}{|c|}{ FEMENINO } \\
\hline & \multicolumn{2}{|c|}{0 a 14} & \multicolumn{2}{|c|}{15 a 29} & \multicolumn{2}{|c|}{30 a 44} & \multicolumn{2}{|c|}{45 a 59} & \multicolumn{2}{|c|}{$>60$} & \multicolumn{2}{|c|}{ Total } \\
\hline Tipos de Accidentes & $N$ & $\%$ & $N$ & $\%$ & $N$ & $\%$ & $N$ & $\%$ & $N$ & $\%$ & $N$ & $\%$ \\
\hline ATT & 120 & 17,5 & 211 & 30,8 & 151 & 22,1 & 98 & 14,3 & 104 & 15,2 & 684 & 47,1 \\
\hline Otras lesiones & 136 & 34,5 & 42 & 10,7 & 29 & 7,4 & 41 & 10,4 & 146 & 37,1 & 394 & 27,1 \\
\hline Suicidios & 9 & 6,9 & 58 & 44,3 & 40 & 30,5 & 17 & 13,0 & 7 & 5,3 & 131 & 9,0 \\
\hline Agresiones & 15 & 9,9 & 77 & 50,7 & 40 & 26,3 & 18 & 11,8 & 2 & 1,3 & 152 & 10,5 \\
\hline
\end{tabular}




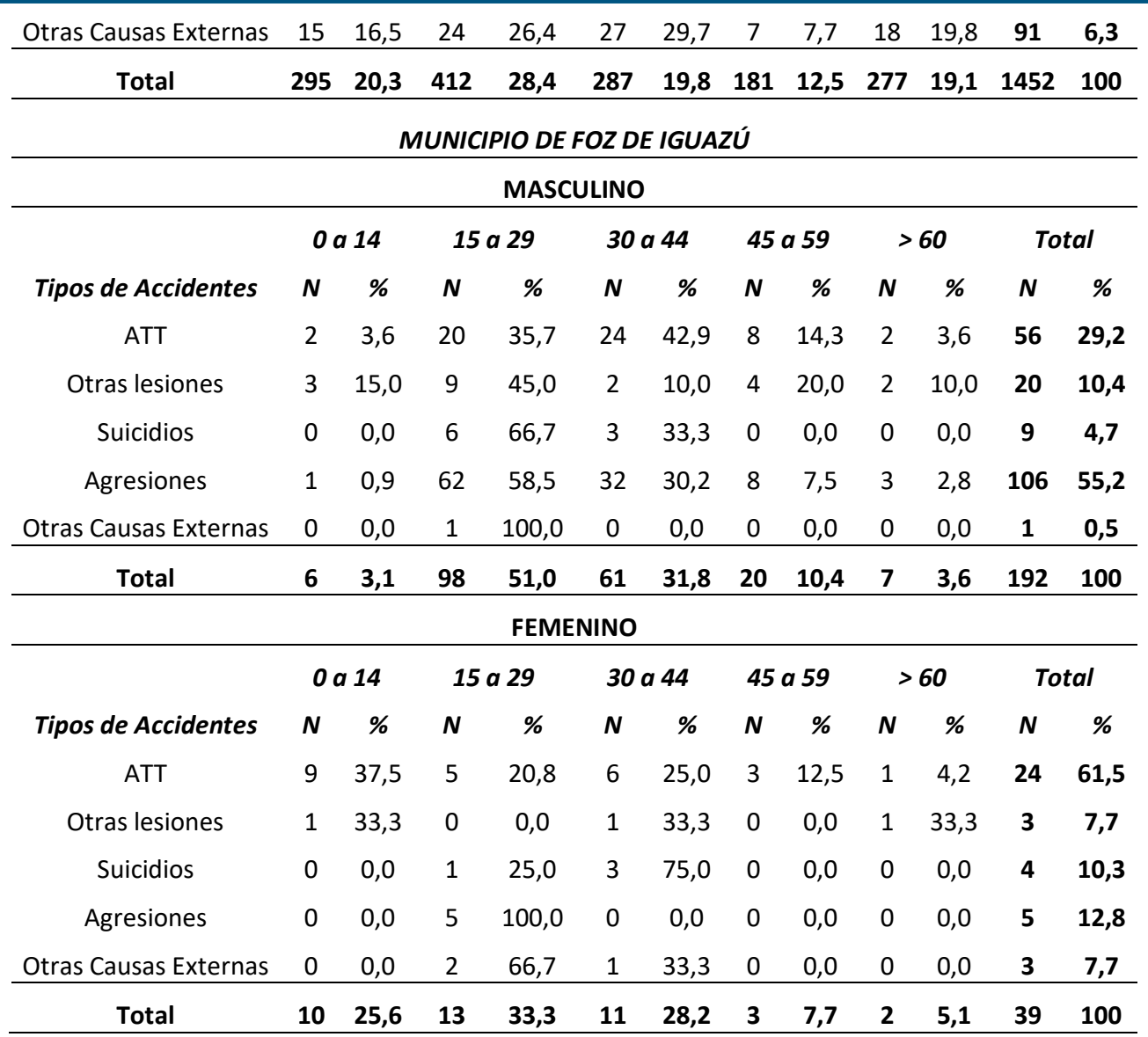

TABLA 4: MORTALIDAD PROPORCIONAL DE GRUPOS DE CAUSAS EXTERNAS POR SEXO Y GRUPO ETARIO, EN EL ESTADO DE PARANÁ Y MUNICIPIO DE FOZ DE IGUAZÚ, 2016.

\begin{tabular}{|c|c|c|c|c|c|c|c|c|c|c|c|c|}
\hline \multicolumn{13}{|c|}{ ESTADO DE PARANÁ } \\
\hline \multicolumn{13}{|c|}{ MASCULINO } \\
\hline Grupo Etario & \multicolumn{2}{|c|}{ o a 14} & \multicolumn{2}{|c|}{15 a 29} & \multicolumn{2}{|c|}{30 a 44} & \multicolumn{2}{|c|}{45 a 59} & \multicolumn{2}{|c|}{$>60$} & \multicolumn{2}{|c|}{ Total } \\
\hline Tipos de Accidentes & $N$ & $\%$ & $N$ & $\%$ & $N$ & $\%$ & $N$ & $\%$ & $N$ & $\%$ & $N$ & $\%$ \\
\hline ATT & 47 & 2,1 & 755 & 34,0 & 604 & 27,2 & 456 & 20,5 & 343 & 15,5 & 2219 & 31,3 \\
\hline Otras lesiones & 79 & 6,4 & 179 & 14,6 & 188 & 15,3 & 230 & 18,7 & 549 & 44,7 & 1229 & 17,3 \\
\hline Suicidios & 2 & 0,3 & 154 & 24,3 & 196 & 30,9 & 162 & 25,5 & 118 & 18,6 & 635 & 9,0 \\
\hline Agresiones & 24 & 0,9 & 1426 & 51,8 & 844 & 30,7 & 303 & 11,0 & 130 & 4,7 & 2753 & 38,8 \\
\hline Otras Causas Externas & 15 & 5,9 & 51 & 20,1 & 76 & 29,9 & 57 & 22,4 & 48 & 18,9 & 254 & 3,6 \\
\hline Total & 167 & 2,4 & 2565 & 36,2 & 1908 & 26,9 & 1208 & 17,0 & 1188 & 16,8 & 7090 & 100 \\
\hline \multicolumn{13}{|c|}{ FEMENINO } \\
\hline & \multicolumn{2}{|c|}{ O $a 14$} & \multicolumn{2}{|c|}{15 a 29} & \multicolumn{2}{|c|}{30 a 44} & \multicolumn{2}{|c|}{45 a 59} & \multicolumn{2}{|c|}{$>60$} & \multicolumn{2}{|c|}{ Total } \\
\hline Tipos de Accidentes & $N$ & $\%$ & $N$ & $\%$ & $N$ & $\%$ & $N$ & $\%$ & $N$ & $\%$ & $N$ & $\%$ \\
\hline ATT & 53 & 9,9 & 151 & 28,3 & 126 & 23,6 & 92 & 17,2 & 112 & 21,0 & 534 & 31,7 \\
\hline Otras lesiones & 53 & 7,2 & 16 & 2,2 & 21 & 2,8 & 42 & 5,7 & 605 & 82,1 & 737 & 43,7 \\
\hline Suicidios & 4 & 3,2 & 19 & 15,2 & 49 & 39,2 & 40 & 32,0 & 13 & 10,4 & 125 & 7,4 \\
\hline Agresiones & 10 & 4,2 & 80 & 33,9 & 80 & 33,9 & 50 & 21,2 & 16 & 6,8 & 236 & 14,0 \\
\hline Otras Causas Externas & 6 & 10,9 & 11 & 20,0 & 12 & 21,8 & 8 & 14,5 & 18 & 32,7 & 55 & 3,3 \\
\hline Total & 126 & 7,5 & 277 & 16,4 & 288 & 17,1 & 232 & 13,8 & 764 & 45,3 & 1687 & 100 \\
\hline
\end{tabular}




\begin{tabular}{|c|c|c|c|c|c|c|c|c|c|c|c|c|}
\hline \multicolumn{13}{|c|}{ MASCULINO } \\
\hline & \multicolumn{2}{|c|}{0 a 14} & \multicolumn{2}{|c|}{15 a 29} & \multicolumn{2}{|c|}{30 a 44} & \multicolumn{2}{|c|}{45 a 59} & \multicolumn{2}{|c|}{$>60$} & \multicolumn{2}{|c|}{ Total } \\
\hline Tipos de Accidentes & $N$ & $\%$ & $N$ & $\%$ & $N$ & $\%$ & $N$ & $\%$ & $N$ & $\%$ & $N$ & $\%$ \\
\hline ATT & 0 & 0,0 & 14 & 31,8 & 10 & 22,7 & 11 & 25,0 & 9 & 20,5 & 44 & 23,4 \\
\hline Otras lesiones & 5 & 18,5 & 1 & 3,7 & 3 & 11,1 & 6 & 22,2 & 12 & 44,4 & 27 & 14,4 \\
\hline Suicidios & 0 & 0,0 & 4 & 30,8 & 4 & 30,8 & 1 & 7,7 & 4 & 30,8 & 13 & 6,9 \\
\hline Agresiones & 2 & 2,2 & 52 & 57,8 & 22 & 24,4 & 11 & 12,2 & 3 & 3,3 & 90 & 47,9 \\
\hline Otras Causas Externas & 5 & 35,7 & 1 & 7,1 & 3 & 21,4 & 2 & 14,3 & 3 & 21,4 & 14 & 7,4 \\
\hline Total & 12 & 6,4 & 72 & 38,3 & 42 & 22,3 & 31 & 16,5 & 31 & 16,5 & 188 & 100 \\
\hline \multicolumn{13}{|c|}{ FEMENINO } \\
\hline & \multicolumn{2}{|c|}{0 a 14} & \multicolumn{2}{|c|}{15 a 29} & \multicolumn{2}{|c|}{30 a 44} & \multicolumn{2}{|c|}{45 a 59} & \multicolumn{2}{|c|}{$>60$} & \multicolumn{2}{|c|}{ Total } \\
\hline Tipos de Accidentes & $N$ & $\%$ & $N$ & $\%$ & $N$ & $\%$ & $N$ & $\%$ & $N$ & $\%$ & $N$ & $\%$ \\
\hline ATT & 3 & 27,3 & 2 & 18,2 & 4 & 36,4 & 2 & 18,2 & 0 & 0,0 & 11 & 25,6 \\
\hline Otras lesiones & 2 & 15,4 & 0 & 0,0 & 1 & 7,7 & 0 & 0,0 & 10 & 76,9 & 13 & 30,2 \\
\hline Suicidios & 0 & 0,0 & 0 & 0,0 & 1 & 50,0 & 1 & 50,0 & 0 & 0,0 & 2 & 4,7 \\
\hline Agresiones & 0 & 0,0 & 1 & 11,1 & 6 & 66,7 & 1 & 11,1 & 1 & 11,1 & 9 & 20,9 \\
\hline Otras Causas Externas & 2 & 25,0 & 1 & 12,5 & 0 & 0,0 & 1 & 12,5 & 4 & 50,0 & 8 & 18,6 \\
\hline Total & 7 & 16,3 & 4 & 9,3 & 12 & 27,9 & 5 & 11,6 & 15 & 34,9 & 43 & 100 \\
\hline
\end{tabular}

\section{DISCUSIÓN}

Las tasas ajustadas por causas externas muestran, entre 1996 y 2016, en el Estado de Paraná una tendencia estable y una tendencia decreciente en el Municipio de Foz Iguazú, pero con magnitudes mayores en el municipio. Con diferencia a nivel nacional en un estudio hecho de análisis de datos de mortalidad podemos observar que las causas externas en el Brasil van en aumento a partir del año 1977 a 1996, en el año 1977 se registró 55.240 víctimas fatales un 49,9 por 100000 habitantes y en 1996107292 muertes 69,8 por 100000 habitantes, en los 18 años de estudio los números de óbitos es siempre creciente revelando un aumento del $40 \%$ prácticamente (9). En otro estudio en Brasil desde el año 1980 al 2004 se puede observar la misma tendencia de aumento en las tasas por CE de 59 para poco más del 70 para cada 100 mil habitantes, lo que representa un incremento de cerca del $20 \%$ (10-12). Este aumento puede estar caracterizado por las faltas de políticas públicas efectivas para tal problema de salud y las faltas de conocimiento con referencias a las causas que determinan las muertes por causas externas. Utilizando la Clasificación Internacional de Enfermedades -CIE -10, el mayor número de óbitos fueron las Enfermedades del Aparato Circulatorio, seguido de neoplasias y causas externas provocando en el año 2005, 127633 víctimas fatales a 145842 en el 2011 (4).
Con relación a este trabajo a la tendencia por sexo en las tasas el sexo masculino tanto como en Paraná y Foz de Iguazú es mayor que con relación a las mujeres, con magnitudes mayores en el municipio. Este perfil se puede atribuir, a la mayor exposición de hombres jóvenes a algunas actividades laborales de riesgo, al consumo de alcohol, a los comportamientos agresivos y a la dirección peligrosa de vehículos automotores (11). El año 2000 en el artículo las Causas Externas en el Brasil, comparando la mortalidad y morbilidad, el coeficiente de mortalidad por causas externas encontrado fue de 69,7 por 100000 habitantes, siendo 119,0 en el sexo masculino y 21,8 en el sexo femenino por 100000 habitantes, siendo una razón los coeficientes masculinos/femenino de 5,5 (12).

Un estudio similar en el 2013 resalta que las CE fueron responsables por 151683 óbitos registrados en el SIM, siendo la mayor parte entre hombres (82,2 \%) y personas con edad entre 20 y 39 años (43,8 \%) (13). Desde el punto de vista de las características de esas víctimas, hemos verificado que el hombre es siempre más vulnerable, visto que las tasas en el sexo masculino corresponden a 3 o 4 veces más de lo que ocurre en mujeres (10). En Minas Gerias los datos confirman que la tendencia de mayor riesgo de óbitos por causas externas es para hombres, con registro de que están 4 veces más sujetos a morir por alguna de las causas externas que las mujeres (14).

Según este estudio en cuanto a la mortalidad 
proporcional de los grupos de causas externas más importantes de los 21 años de estudio, según figura 3 y 4 se observa que en el Estado de Paraná la primera causa de muerte con el $36,8 \%$ corresponde a ATT, agresiones $32,6 \%$ y otros tipos de accidentes $18,8 \%$, del total de los óbitos del periodo estudiado. Con relación al Municipio de Foz de Iguazú las agresiones representan la primera causa de muerte con el $56,3 \%$, ATT el $25,5 \%$ y otros tipos de accidentes 10,9 de los óbitos. Se verificó, por ejemplo, que muchas víctimas de ese tipo de evento buscaron atención en más de un servicio de salud. La proporción de atención previa por la misma causa externa varió de 6,6 \% en Boa Vista/RR a 50,6 \% en Victória/ES. En la atención por accidentes, la proporción de eventos relacionados al trabajo (accidentes de trabajo) varió de $21,2 \%$ en Macapá/AP a $44 \%$ en Curitiba/PR. Para el total de atenciones (accidentes y violencias), esa proporción varió de 9,5\% en Macapá/AP a 24,9\% en Salvador/BA. Otro dato importante fue la proporción de ingestión de bebida alcohólica en las seis horas anteriores al evento (1).

A partir del coeficiente de regresión de los grupos de CE en los años de estudios se observa que en el Estado de Paraná tanto como en el Municipio de Foz de Iguazú, los casos de accidentes de tránsitos han disminuido, con relación a otros tipos de accidentes en Paraná la tendencia disminuye, con diferencia de Foz de Iguazú que está en aumento. Agresiones en el Estado de Paraná aumenta con diferencia de Foz de Iguazú que disminuye. Suicidios y Otras causas externas en Paraná está en disminución, con relación de Foz de Iguazú que va en aumento.

Un estudio similar, las agresiones permanecieron como la causa específica responsable por la mayor tasa de mortalidad, sin gran variación a lo largo del período. A seguir, las tasas de mortalidad por ATT ocuparon la segunda posición entre las tasas de mortalidad por causas externas, presentando evolución creciente $(25,7 \%$ de 2000 a 2013), especialmente a partir de 2010, aunque con las menores tasas de mortalidad, las caídas y lesiones autoprovocadas presentaron una evolución también creciente en el período, con un aumento de $148 \%$ y $33,3 \%$, respectivamente, entre 2000 y 2013 (6).

En relación a las demás causas externas, estas incluyen caídas, exposición a fuerzas mecánicas, ahogamientos y sumersión por accidentes, riegos accidentales a respiración, exposición a corrientes eléctricas. Humos, fuego, contacto con fuentes de calor y sustancias calientes, exposición a fuerzas de la naturaleza, envenenamiento e intoxicación, entre otras causas. En este grupo de causas, las caídas representan las principales causas de óbitos siendo el más relevante en este estudio. Estudio realizado en Minas Gerias en 2010 sobre causas externas en personas de la tercera edad registro el incremento de óbitos por causas externas por caídas, siendo principalmente debido a fracturas diversas y traumatismo cráneo encefálico (14).

Según el Ministerio de Salud Brasilero, en 2013, la tasa de internación hospitalaria por causas externas fue de 52,6 internaciones por 10 mil habitantes, variando de 31,2 internaciones por 10 mil mujeres a 74,5 internaciones por 10 mil hombres (razón de tasas entre los sexos $=2,4)(6)$, esa diferencia puede estar relacionada a las diferencias de comportamiento de cada sexo y a los factores culturales que determinan mayor libertad a los hombres $(15,16)$.

Un estudio realizado en el Estado de San Paulo, municipio de José de los Campos, el mayor gasto medio de internaciones en pacientes con lesiones ocasionadas por agresiones y accidentes de transporte, en relación a las mismas lesiones por otras causas, puede significar en mayores costos con el tratamiento de algunos traumas por tener una consecuencia mayor en la gravedad de las lesiones en ese tipo de causas externas, lo que acarrea la realización de procedimientos de mayor complejidad (17). En el artículo impacto económico de las causas externas en Brasil según lunes se puede observar claramente que las hospitalizaciones provenientes de causas externas representan una proporción mayor de días de UTI que del total de las otras internaciones (18). Brasil ha revelado la mayor desigualdad de mortalidad entre hombres y mujeres en lo que se habla de causas externas, Esa desigualad de mortalidad está asociada al comportamiento social y cultural de la población masculina que se expone mayormente a los riesgos (19).

El presente estudio demostró que las agresiones representan en de las causas más importantes de muerte supera el 54,6\% de las muertes, con mayor índice en el sexo masculino $90 \%$ de los casos y mujeres $10 \%$, en edades de 15 a 29 años de edad. De acuerdo con el mapa de violencias de los Municipios de Brasil, en 2004 Paraná ocupaba el décimo primer lugar en la clasificación general de los Estados en la mortalidad por homicidios, en cuanto a 10 años atrás, en 1994 estaba en la posición décima sexta (20). El Ministerio de Salud de Brasil los homicidios son un tema prácticamente ligado al género, es lo que muestran los datos de 2013, los homicidios ocurrieron, principalmente en personas del sexo masculino $(91,7$ $\%)$. La razón entre las tasas de homicidios de hombres 
y mujeres fue de 11,2 veces. El análisis de los datos señala el homicidio como un mal social fuertemente vinculado al sexo masculino. La literatura ha llamado la atención por el comportamiento agresivo y arriesgado de los hombres explicando la mayor incidencia de muertes por homicidio en comparación con las mujeres (6).

En los 21 años de estudios fueron registrados 63170 (37\%) óbitos de accidentes terrestres en el Estado Paraná. En cambio, en el municipio de Foz de Iguazú se registraron 1755 (26,3\%), con mayores magnitudes para los dos locales en el sexo masculino $80 \%$ en jóvenes adultos de ( 20 a 45 años) y en mujeres $20 \%$. El municipio de Foz de Iguazú está localizada en una de las fronteras más movidas del Brasil, más conocida como la triple frontera, y no solo por ser la tierra de las Cataras del Iguazú, sino por el libre tránsito con la República del Paraguay - Ciudad del Este uno de los centro de compras más importantes de la región según el periódico Radio Cultura Foz 278969 pasaron caminando por el puente de la amistad, en el 2016 la media de vehículos que paso por el puente fue de 29 906 vehículos al día siendo $40 \%$ de motos y $36,2 \%$ de autos particulares.

Según la Organización Panamericana de la Salud (2016), en el 2013 la tasa promedio de mortalidad por accidentes fue de 15,9 por 100000 habitantes en la región de las Américas, siendo con la mayor incidencia República Dominicana al 30 por 100.000 habitantes, Belice con 25,0 y Brasil con 22,5 por 100000 habitantes superando grandemente la media regional de 15,9 por 100000 habitantes, cifra inferior a la tasa mundial de 17,4. Sin embargo, tras este promedio regional se ocultan marcadas diferencias de un país a otro, ya que las tasas nacionales varían mucho (21).

Uno de los factores de riesgo más predominantes para las CE es el consumo de alcohol principalmente accidentes de tránsitos terrestres y agresiones, el uso de alcohol es considerado como una causa posible que influye a que tales problemas de salud sigan incrementando por la facilidad del acceso a la compra de bebidas alcohólicos. Considerando las diferentes sustancias psicoactivas, el uso indebido del alcohol tiene una mayor prevalencia global, trayendo graves consecuencias para la salud pública mundial siendo responsable del $1,5 \%$ de todas las muertes a nivel mundial y el 2,5\% del total de años perdidos ajustados por incapacidad (22). Los efectos inmediatos del uso de alcohol tienen impacto en casi todos los tipos de accidentes debido a la reducción de la habilidad psicomotora. La ingesta de elevadas cantidades aumenta el riesgo exponencialmente, el uso pesado en una única ocasión también tiene importante relación con suicidio y violencia (23).

\section{CONTRIBUCIÓN DE LOS AUTORES}

DGADZ: Concepción y diseño del estudio, procesamiento y análisis de datos, redacción artículo, revisión de la literatura, revisión crítica del artículo y aprobación versión final. CJG: Procesamiento y análisis de datos, redacción del artículo, revisión crítica del artículo y aprobación versión final.

\section{REFERENCIAS}

1. Akemi M, Maffei De Andrade S, Soares D, Freitas T, Marques M, Pelissari D, et al. Cualidad de la información sobre causas externas en el sistema de Informaciones Hospitalares. Rev. Saude Publica. 2009; 43(3):413-420. https://doi.org/10.1590/S0034-8910200900300004

2. Araujo E, Nascimento M, Fernandes N, Santos F, Lima Mauricio, Hogan $V$, et al. Distribuição espacial da mortalidade por homicídio e desigualdades sociais segundo a raça/cor em um espaço intra-urbano no Brasil. Rev. Bras Epidemiol. 2010;13(4), 549-560. https://doi.org/10.1590/S1415-790X2010000400001

3. Bacchieri G, Barros A. Acidentes de trânsito no Brasil de 1998 a 2010: Muitas mudanças e poucos resultados. Rev. Saude Publica. 2011; 45(5), 949-963. https://doi.org/10.1590/S0034-89102011005000069

4. Bastos Y, Andrade S, Soares D. Características dos acidentes de trânsito e das vítimas atendidas em serviço préhospitalar em cidade do Sul do Brasil, 1997/2000. Cad. Saude Public. 2005; 21(3), 815-822. https://doi.org/10.1590/S0102-311X2005000300015

5. Blanski G, Vieira de Souza J, Lenardt M, Pesck R, Seima M, De Oliveira Borges P. Caracterização de idosos vítimas de acidentes por causas externas. Rev. Cogitare Enferm. 2014; 19(3), 506-513. URL.

6. Brasil. Ministerio de Salud de Brasil. Secretaría de Vigilancia en salud. Salud Brasil 2014 - Un Análisis de la Morbimortalidad por Causas Externas. Brasilia: Ministerio de salud de Brasil, 2015. URL.

7. Rodrigues $M$, Reis von Doellinger V, Periera L, Santos $M$, Pimental T, Mendes J. Diferenciais de morbimortalidade por causas externas: resultados do estudo Carga Global de Doenças no Brasil, 2008. Cad. Saude Publica. 2015; 31(1), 117. https://doi.org/10.1590/0102-311X00191113

8. Cardona D, Peláez E, Aidar T, Ribotta B, Álvarez M. Mortalidad por causas externas en tres ciudades latinoamericanas: Córdoba (Argentina), Campinas (Brasil) y Medellín (Colombia), 1980-2005. Rev Bras Estud Popul. 2008; 25(2), 335-352. https://doi.org/10.1590/5010230982008000200009

9. Cherpitel C. J. Focus on: the Burden of Alcohol use trauma and Emergency outcomes, Alcohol RES. 2014; 35(2). 150-154. URL.

10. Corassa R, Falci D, Gontijo C, Machado G, Alves P. Evolução da mortalidade por causas externas em Diamantina (MG), 2001 a 2012. Cad. Saúde Colet. 2017; 25(3), 302-314. https://doi.org/10.1590/1414462×201700030258

11. De Andrade-Barbosa T, Xavier-Gomes L, AndradeBarbosa V, Caldeira, A. Male mortality due to external causes in the State of Minas Gerais, Brazil. Ciencia e Saude Coletiva. 2013; 18(3), 711-719. https://doi.org/10.1590/S1413-81232013000300017 
12. Alves-Junior E, Lima-Paula F. La prevención de caídas bajo el aspecto de la promoción de la salud. Fitness \& Performance, 2008; 7(2), 123-129. URL.

13. Denepotti V, Xavier-Gomes L, De Andrade T. Tendencia da Da Mortalidade por Homicídio em Foz do Iguaçu e Paraná, 2010 a 2015. Rev. Bras. de Iniciação Científica. 2018; 5(2), 222-234. URL.

14. Di Nubila H, Buchalla C. O papel das Classificações da OMS - CID e CIF nas definições de deficiência e incapacidade. Rev Bras Epidemiol. 2008; 11(2), 324335. https://doi.org/10.1590/S1415-790X2008000200014

15. Rosa T. Benicio-D’Aquino M, Latorre M, Ramos L. Fatores determinantes da capacidade funcional entre idosos. Rev. Saúde Pública. 2003; 37(1), 40-48. https://doi.org/10.1590/S0034-89102003000100008

16. Gawryszewski V, Koizumi M, Mello-Jorge M. As causas externas no Brasil no ano 2000: comparando a mortalidade e a morbidade. Cad. Saúde Pública, 2004; 20(4), 995-1003. http://dx.doi.org/10.1590/S0102-311X2004000400014

17. Golias A, Caetano R. Acidentes entre motocicletas: análise dos casos ocorridos no estado do Paraná entre julho de 2010 e junho de 2011. Ciência \& Saúde Coletiva. 2013; 18(5), 1235-1246. https://doi.org/10.1590/S141381232013000500008

18. Texeira-Gonsaga R, Fernandes C, Araujo E, Scaramucci F, Tadao M, Bolini M. Avaliação da mortalidade por causas externas. Rev. Col Bras Cir. 2012; 39(4), 263-267. http://dx.doi.org/10.1590/S0100-69912012000400004

19. lunes R. III - Impacto econômico das causas externas no Brasil: Um esforço de mensuração. Revista de Saude Publica. 1997; 31(4), 38-46. http://dx.doi.org/10.1590/\$0034-89101997000500004

20. Jorge M, Gawryszewski V, Latorre M. I - Análise dos dados de mortalidade. Rev. Saúde Pública. 1997; 31(4), 5-25. http://dx.doi.org/10.1590/S0034-89101997000500002

21. Alves A, Duarte I, Lins L, Sales M, Rocha S, Cavalcanti A. Mortalidade por causas externas nos municípios de Arapiraca (al) e Mossoró (rn) - 1999-2008. Rev. Baiana de Saude Publica. 2012; 36(1), 134-147. URL.

22. Mercy J, Zwi A, Lozano R. Books \& Electronic Media. World Health Organization, 84. 2016 2006. URL.

23. Niwa, H.. Paraná Projeções de População 1991 - 2020. IPARDES. 1991; 134(4), 635-646. URL. 